\title{
Meningoencefalite tuberculosa: avaliação de 231 casos
}

\author{
Tuberculosis meningoencephalitis: exposure of 231 cases \\ Ceuci Nunes, Sérgio Cunha, Nilton Gomes, Adelci Tavares, \\ Dilcinéia Amorim, Irênio Gomes e Ailton Melo
}

\begin{abstract}
Resumo Neste estudo foram avaliados 231 pacientes com meningoencefalite tuberculosa, sendo que 62 casos tiveram diagnóstico comprovado e 169 apresentavam quadro clínico e laboratorial compatíveis com este diagnóstico. Foram 127 (55\%) pacientes do sexo masculino, a idade variou de 1 mês a 68 anos, com 97 (42\%) na faixa etária igual ou inferior a um ano. As características clínicas, demográficas e liquóricas foram estudadas e comparadas entre os casos confirmados e os de diagnóstico provável. Em conclusão reafirmamos a gravidade desta doença, com altas taxas de letalidade principalmente na faixa etária de zero a quatro anos e a possibilidade de erros diagnósticos nas apresentações com formas agudas e predominância de neutrófilos no líquor.
\end{abstract}

Palavras-chaves: Meningoencefalite tuberculosa. Meningite tuberculosa. Tuberculose do SNC. Tuberculose.

Abstract This study assessed 231 cases of tuberculous meningitis of which 62 (26.8\%) had diagnostic confirmation against 169 (73.2\%) with only clinical picture and laboratorial indication for this diagnosis. Fifty-five percent of the sample was male; ages ranged from one month to 68 years, $42 \%$ comprising children below four years. Clinical, demographic and liquoric characteristics were investigated and compared amongst those with likely and confirmed diagnosis. In conclusion, atention is drawn to the severity of this desease with high rates of lethality mainly within the agerange of 0-4 years, and to the possibility of misdiagnosis in the presentation of acute forms and predominance of neutrophils in the liquor.

Key-words: Tuberculous meningoencephalitis. Tuberculous meningitis. CNS tuberculosis. Tuberculosis.

Hospital Couto Maia, Secretaria da Saúde do Estado da Bahia e Faculdade de Medicina da Universidade Federal da Bahia, Salvador, BA. Endereço para correspondência: Dra. Ceuci Nunes. Centro Odonto-Médico Henri Dunnant. R. Agnelo de Brito 187 sala 214, Garibaldi. 41760-100 Salvador, BA.

Telefax: (071) 247-8233.

Recebido para publicação em 06/10/97. 
A reemergência 10 da tuberculose neste final de século trás no seu contexto além do aumento das formas multiresistentes 7 , o possível aumento das formas extrapulmonares, dentre elas a meningoencefalite. Esta forma clínica é a mais grave12, tem alta taxa de letalidade, até $30,0 \%$ de sequelas neurológicas graves e incapacitantes 17 e sua freqüência é relacionada à incidência da forma pulmonar bacilífera1. Dados da Secretaria da Saúde de Estado da Bahia mostram que em 1995 foram registrados 8819 casos novos confirmados de tuberculose, com coeficiente de incidência de 70,3 por 100.000 habitantes e 40 casos de meningoencefalite tuberculosa, com coeficiente de incidência de 0,3 por 100.000 habitantes. A letalidade mostrada em um estudo do Ministério da Saúde, realizado em 1983 , foi de $44,7 \%$ na faixa etária de 0-4 anos 16 .

O quadro clínico da meningoencefalite tuberculosa é variável de acordo com a faixa etária 26 , sendo classicamente dividido em três estágios 22 de acordo com sintomas e sinais mais ou menos específicos e a gravidade do comprometimento neurológico9.

A dificuldade diagnóstica da meningoencefalite tuberculosa é descrita por diversos autores 24 . O método mais simples e exeqüível de diagnóstico, a pesquisa direta do bacilo pela técnica de Ziehl-Nielsen ${ }^{3}$, tem baixa sensibilidade: a positividade varia de 10 a 40\% 26 e no Brasil foram observadas taxas ainda menores, de até de 4,8\%20. A cultura do Mycobacterium tuberculosis, apesar de representar a prova-ouro de confirmação diagnóstica, tem a grande limitação do tempo necessário para o crescimento do bacilo, que chega a 45 dias $^{3}$. As técnicas mais modernas de confirmação diagnóstica, como a PCR (polymerase chain reaction), tem ainda o seu uso limitado a laboratórios de pesquisa clínica, não estando disponíveis na prática clínica diária6.

Com todos os problemas enfrentados pelas equipes médicas para esclarecimento diagnóstico destes pacientes, o que vemos com freqüência é o diagnóstico baseado no quadro clínico e na avaliação liquórica 14 .

Deste modo nosso objetivo foi o de conhecer melhor a população de pacientes com meningoencefalite tuberculosa internados no Hospital Couto Maia em Salvador, referência para doenças infecto-contagiosas no Estado, e rever os critérios diagnósticos utilizados.

\section{MATERIAL E MÉTODOS}

Neste estudo descritivo, retrospectivo, foi realizada a análise comparativa dos casos com meningoencefalite tuberculosa confirmada com os casos onde este diagnóstico foi presuntivo.

Estes pacientes foram retirados de três bancos de dados preexistentes com 2608 pacientes, todos internados no Hospital Couto Maia nos seguintes períodos: ano de 1983, janeiro de 1990 a dezembro de 1992 e março a dezembro de 1994. Os dados colhidos em 1983, objetivaram a participação em um estudo sobre meningoencefalite tuberculosa conduzido pelo Ministério da Saúde em oito estados brasileiros. Os demais dados foram colhidos para estudos das meningites em geral. No total foram revistos os dados de prontuários de 231 pacientes. Deste estudo foram excluídos os pacientes com as características descritas a seguir, com o objetivo de eliminar as meningites de etiologia não tuberculosa: cultura positiva no líquor ou no sangue para bactérias ou fungos; presença de bactérias no líquor pelo método de Gram; tinta da China positiva no LCR; celularidade no LCR maior que 2000 células; celularidade maior que 1000 células com predominância de neutrófilos; pacientes que não usaram antibióticos nem esquema tríplice tuberculostático e que receberam alta e pacientes transferidos. Após esta seleção foram aplicados os seguintes critérios de inclusão: pacientes com diagnóstico confirmado de meningoencefalite tuberculosa (baciloscopia e/ou cultura), técnica de PCR ou exame anátomo- patológico pós-mortis; pacientes com diagnóstico liquórico de meningite com as seguintes características: celularidade $\geq 10$ a $\leq 1000$ células, com predominância de linfócitos e proteinorraquia $\geq 60 \mathrm{mg} \%$.

Após esta seleção, foram revistos as características demográficas, clínicas e laboratoriais de todos os pacientes. $\mathrm{Na}$ comparação entre as características dos casos confirmados e prováveis utilizou-se o teste quiquadrado ou o teste exato de Fisher para variáveis categóricas, e o teste não-paramétrico de Mann-Whitney para variáveis contínuas ou discretas. A análise estatística foi realizada pelo EPI INFO versão 6 e a diferença foi considerada significativa quando $p<0,05$.

\section{RESULTADOS}

Foram estudados 231 pacientes sendo 127 $(55,0 \%)$ do sexo masculino e $104(45,0 \%)$ do 
sexo feminino. As idades variaram de menos de 1 mês a 68 anos; $97(42,0 \%)$ pacientes tinham idade igual ou inferior a um ano. A evolução dos casos estudados de acordo com a faixa etária foi analisada utilizando-se o teste qui-quadrado de tendência mostrando uma concentração tanto do número de casos como do número de óbitos na faixa de zero a quatro anos (Tabela 1).

Tabela 1- Evolução dos 231 casos de meningoencefalite tuberculosa de acordo com a faixa etária.

\begin{tabular}{|c|c|c|c|c|c|c|}
\hline \multirow{2}{*}{$\begin{array}{c}\text { Faixa etária } \\
\text { (anos) }\end{array}$} & \multicolumn{2}{|c|}{ Alta } & \multicolumn{2}{|c|}{ óbito } & \multicolumn{2}{|c|}{ Total } \\
\hline & $\mathrm{n}^{\circ}$ & $\%$ & $\mathrm{n}^{\circ}$ & $\%$ & $\mathrm{n}^{\circ}$ & $\%$ \\
\hline$\overline{0 \mid-4}$ & 23 & 23,7 & 74 & 76,3 & 97 & 42 \\
\hline $5|-| 14$ & 29 & 63,0 & 17 & 36,9 & 46 & 19,5 \\
\hline $15|-| 24$ & 13 & 43,3 & 17 & 56,6 & 30 & 13 \\
\hline $25|-| 34$ & 18 & 62,1 & 11 & 37,9 & 29 & 12,6 \\
\hline $35|-| 44$ & 6 & 33,3 & 12 & 66,6 & 18 & 7,8 \\
\hline $45|-| 55$ & 4 & 66,6 & 2 & 33,3 & 6 & 2,6 \\
\hline$>55$ & 3 & 60,0 & 2 & 40,0 & 5 & 2,2 \\
\hline Total & 96 & 41,6 & 135 & 58,4 & 231 & 100 \\
\hline
\end{tabular}

$\chi^{2}$ Tendência: $9,007 p=0,008$

O tempo de doença variou de zero (paciente chegou ao hospital no mesmo dia do início dos sintomas) a 90 dias, sendo a média de 10,71 dias e o desvio padrão de 11,57 . Os sinais e sintomas mais freqüentes estão descritos na Tabela 2.
As características do exame do LCR no que diz respeito à celularidade, dosagem de proteína e de glicose, podem ser observadas na Tabela 3.

Dos 231 casos, $146(63,2 \%)$ usaram o esquema específico para tuberculose e 68

Tabela 2- Frequência dos mais importantes sinais e sintomas em 231 casos de meningoencefalite tuberculosa.

\begin{tabular}{|c|c|c|c|}
\hline Sinal ou sintoma & Presença & № de casos & $\%$ \\
\hline Rigidez de nuca & 176 & 207 & 85,0 \\
\hline Febre & 173 & 213 & 81,2 \\
\hline Alteração do nível de consciência & 164 & 207 & 79,2 \\
\hline Convulsão & 77 & 160 & 48,1 \\
\hline Alteração nervos cranianos & 48 & 101 & 47,5 \\
\hline Cefaléia & 55 & 117 & 47,0 \\
\hline Outras alterações neurológicas & 30 & 95 & 31,6 \\
\hline Hemiparesias & 9 & 124 & 7,3 \\
\hline
\end{tabular}

Tabela 3- Características do exame do LCR em 231 casos de meningoencefalite tuberculosa.

\begin{tabular}{lccc}
\hline Características do LCR & Média \pm Desvio padrão & Faixa & Anormalidade (\%) \\
\hline Celularidade $\left(\mathrm{mm}^{3}\right)$ & $288,32 \pm 270,85$ & 4 a 1549 & 100 \\
Proteína $(\mathrm{mg} \%)$ & $182,47 \pm 101,08$ & 30 a 500 & 98,4 \\
Glicose $(\mathrm{mg} \%)$ & $42 \pm 13,2$ & 20 a 75 & 50,9 \\
\hline
\end{tabular}

$(29,4 \%)$ não, sendo que em $17(7,4 \%)$ não foi possível a obtenção deste dado. Quanto ao uso de corticóide, o mesmo foi utilizado em 114 $(49,4 \%)$ casos, não utilizado em $78(33,8 \%)$ e em $39(16,8 \%)$ não foi possível a obtenção do dado.
A confirmação diagnóstica ocorreu em 62 casos, correspondentes a $26,8 \%$ da amostra, com cultura em 35 casos, exame anátomopatológico pós-mortis em 25, PCR em 12 e baciloscopia do líquor em três. Em 13 casos houve confirmação por mais de uma forma. 
Como mostrado na Tabela 4, a média de idade dos casos com diagnóstico confirmado foi maior do que nos casos com diagnóstico provável $(p<0,02)$. No conjunto dos casos de cada grupo, a glicorraquia foi mais baixa $(p<0,0001)$ e a taxa de letalidade foi maior $(p<0,02)$ nos casos confirmados, quando comparados aos prováveis. As demais características estudadas tiveram freqüências em médias semelhantes $(p>0,005)$ nos dois grupos avaliados.

\begin{tabular}{|c|c|c|c|}
\hline Características & $\begin{array}{c}\text { Comprovados } \\
62(26,8 \%)\end{array}$ & $\begin{array}{c}\text { Prováveis } \\
169(73,2 \%)\end{array}$ & Teste $(p)$ \\
\hline \multicolumn{4}{|l|}{ Sexo n $(\%$} \\
\hline feminino & $33(53,2 \%)$ & $71(42 \%)$ & $2,308^{*}(0,126)$ \\
\hline masculino & $29(46,8 \%)$ & $98(58 \%)$ & \\
\hline Idade (meses) (média ( desvio padrão) & $7,29 \pm 2,79$ & $5,24 \pm 3,4$ & $5,626^{\star *}(0,017)$ \\
\hline Idade (anos) (média ( desvio padrão) & $13,73 \pm 15,20$ & $18,49 \pm 15,37$ & $3,486^{\star *}(0,062)$ \\
\hline Tempo de doença (média ( desvio padrão) & $13,33 \pm 14,76$ & $9,74 \pm 9,64$ & $3,77^{\star *}(0,052)$ \\
\hline \multicolumn{4}{|l|}{ Febre- n(\%) } \\
\hline presente & $53(88,3 \%)$ & $120(78,4 \%)$ & $2,77^{\star}(0,096)$ \\
\hline ausente & $7(11,9 \%)$ & $33(21,6 \%)$ & \\
\hline \multicolumn{4}{|l|}{ Cefaléia n(\%) } \\
\hline presente & $23(46,0 \%)$ & $32(47,8 \%)$ & $0,04^{*}(0,850)$ \\
\hline Ausente & $27(54,0 \%)$ & $35(52,2 \%)$ & \\
\hline \multicolumn{4}{|l|}{ Rigidez de nuca n(\%) } \\
\hline presente & $46(82,1 \%)$ & $130(86,1 \%)$ & $0,50^{\star}(0,479)$ \\
\hline ausente & $10(17,8 \%)$ & $21(13,9 \%)$ & \\
\hline \multicolumn{4}{|l|}{ Convulsão n(\%) } \\
\hline presente & $25(44,6 \%)$ & $52(47,3 \%)$ & $0,42^{*}(0,517)$ \\
\hline Ausente & $31(55,4 \%)$ & $52(47,3 \%)$ & \\
\hline \multicolumn{4}{|l|}{ Alteração consciência: nº (\%) } \\
\hline leve & $5(9,4 \%)$ & $62(55,9 \%)$ & $49(44,1 \%)$ \\
\hline moderada/grave & $48(90,6 \%)$ & $3,33^{*}(0,067)$ & \\
\hline Glicose no LCR (média ( desvio padrão) & $37,56 \pm 12,51$ & $44,21 \pm 12,78$ & $13,19^{*}(0,001)$ \\
\hline Proteína no LCR (média ( desvio padrão) & $185,34 \pm 105,77$ & $175,85 \pm 91,02$ & $0,21^{*}(0,643)$ \\
\hline Celularidade no LCR (média ( desvio padrão) & $305,42 \pm 224,71$ & $247,31 \pm 212,77$ & $4,93^{*}(0,26)$ \\
\hline \multicolumn{4}{|l|}{ Evolução nº (\%) } \\
\hline óbito & $46(74,2 \%)$ & $89(52,7 \%)$ & $5,82^{\star}(0,015)$ \\
\hline Alta & $16(25,8 \%)$ & $80(47,3 \%)$ & \\
\hline
\end{tabular}

* Qui-quadrado ou teste de Fischer

** Teste não- paramétrico de Mann-Whitne

Para possibilitar uma melhor comparação e homogeinizar a amostra foram retirados os valores extremos observados nos casos confirmados de proteinorraquia (três casos com valores $\geq 500$ ) e de celularidade (quatro casos acima de 1000 células). Na avaliação dos casos confirmados onde não ocorreu seleção quanto às características liquóricas, observamos a 
predominância de células polimorfonucleares em $13(21,3 \%)$ casos. Nestes casos o tempo de doença variou de um a 19 dias, com média de 9,1 e desvio padrão de 5,8 .

\section{DISCUSSÃO}

Este estudo reafirma a alta letalidade da meningoencefalite tuberculosa, refletindo a gravidade desta doença em nosso meio. A taxa encontrada é semelhante à observada na Bahia em $1985(55,3 \%)^{16}$ e no Egito em 1993 $(54,7 \%)^{11}$. A manutenção destas altas taxas de letalidade na Bahia mostra que a evolução da medicina nos últimos 10 anos, não trouxe benefícios a esses pacientes. Esta letalidade é maior nos menores de cinco anos, faixa etária mais acometida pela doença, principalmente menores de um ano ${ }^{2}$, como também mostram outros autores23.

Entre as características clínicas, observouse que grande percentagem dos casos (42\%) chegavam ao hospital em coma. Outros dois estudos realizados na Índia mostraram percentuais de 46,423 e 43\%8, enquanto no sudoeste dos Estados Unidos foi encontrada uma taxa de $19 \%$ de pacientes admitidos no estágio III da doença e sem presença de coma8. Estes dados refletem, provavelmente, a maior gravidade desta doença nos países em desenvolvimento que possuem em comum algumas características como a precariedade dos serviços de saúde, piores condições de vida que levam a maior disseminação do bacilo e maior gravidade dos casos.

O tempo de doença é inferior ou igual a sete dias em $47,3 \%$ dos casos, chamando a atenção de que nem sempre, contrariando o descrito na maioria da literatura estudada a meningoencefalite tuberculosa é subaguda ou crônica15. A presença nesta amostra de número elevado de menores de quatro anos, que nem sempre sabem precisar a sintomatologia, e o contato domiciliar com pacientes bacilíferos ${ }^{18}$ com conseqüente maior carga bacilar, podem justificar a evolução mais rápida e mais grave destes pacientes.

Das características liquóricas, vale a pena destacar a presença de $21,3 \%$ de casos com predominância neutrofílica, numa meningite tida como linfomonocitária. Este dado é descrito por outros autores 9 e pode ser um fator de confusão para o diagnóstico, principalmente se ocorrer em associação com um tempo curto de doença, fazendo-se necessário o diagnóstico diferencial com as meningites chamadas piogênicas.
Este estudo reafirma o que mostra a literatura referente ao diagnóstico etiológico da meningoencefalite tuberculosa. A taxa de confirmação de $26,8 \%$ é semelhante à verificada por outros autores. Chama a atenção o percentual de $1,8 \%$ de positividade da baciloscopia no líquor, taxa similar à observada por Azambuja e cols. em outro estudo brasileiro1. Este exame por ser de baixo custo, fácil execução e resultado rápido poderia ser melhor aproveitado em nosso meio3.

$\mathrm{Na}$ comparação dos pacientes que tiveram diagnóstico comprovado com aqueles de diagnóstico presuntivo, observamos similaridade na maioria das características nos dois grupos. Com exceção da glicorraquia, que foi menor nos casos confirmados e da média de idade em pacientes com idade inferior a um ano, todas as outras características avaliadas foram semelhantes. A hipótese por nós levantada para justificar a diferença da glicose, é que a possibilidade de confirmação diagnóstica pressupõe uma maior quantidade de bacilos, que poderia está levando a um maior consumo de glicose. Entretanto, em relação a faixa etária, nada pudemos observar de sua relação com a confirmação diagnóstica.

Este estudo também serve para confirmar a gravidade de uma doença passível de prevenção, pois a vacina BCG25 comprovadamente reduz a freqüência das formas graves da tuberculose 5 , dentre elas a meningoencefalite 21 . Uma outra forma de prevenção é a busca ativa e tratamento precoce dos casos 4 . Desta forma, o aumento da cobertura vacinal e a retomada do amplo controle de tratamento e de comunicantes poderá diminuir a taxa de incidência de formas bacilíferas e consequentemente da meningoencefalite tuberculosa.

\section{AGRADECIMENTOS}

Ao Hospital Couto Maia integrante da rede hospitalar da Secretaria de Saúde do Estado da Bahia, que pela organização do seu Serviço de Arquivo Médico (SAME) nos permitiu a revisão dos dados de prontuários.

\section{REFERÊNCIAS BIBLIOGRÁFICAS}

1. Azambuja HCP, Picon PD, Rizzon CFC, Coutinho M. Meningite tuberculosa. In: Picon PD, Rizzon CFC, Ott WP (eds) Tuberculose: epidemiologia diagnóstico e tratamento em clínica e Saúde Pública, Rio de Janeiro, p. 433-452, 1993. 
2. Berman S, Kibel MA, Fouril PB, Strebel PM. Childhood tuberculosis and tuberculous meningitis: high incidence rates in the Western Cape of South Africa. Tubercle and Lung Disease 73:349-355, 1992.

3. Brasil. Ministério da Saúde. Controle da Tuberculose: uma proposta de integração ensino-serviço, 3a. edição rev, CNTC/NUTES, Rio de Janeiro, 155 p., 1992.

4. Brasil. Ministério da Saúde. Manual de Normas para o Controle da Tuberculose. Centro Nacional de Epidemiologia, Fundação Nacional de Saúde, Ministério da Saúde, 1995.

5. Camargos PAM, Guimarães MDC, Antunes CMF. Risk Assessment for Acquiring Meningitis Tuberculosis among Children Not Vaccinated with BCG: A CaseControl Study. International Journal of Epidemiology 17:193-197, 1988.

6. Chandramuk A, Allen PRJ, Keen M, Ivanyi J. Detection of Mycobacterial Antigen and Antibodies in the Cerebospinal Fluid of Patients with Tuberculous Meningitis. Journal of Medical Miicrobiology 28:239247, 1985.

7. Cockerill RF, Williams DE, Eisenach KD, Kline BC, Miller LK, Stockman L, Voyles J, Caron GM, Bundy SK, Roberts GD, Wilson WR, Whelen AC, Hunt JM, Persing $\mathrm{DH}$. Prospective Evaluation of the Utility of Molecular Techniques for Diagnosing Nosocomial Tranmission of Multidrug-Resistant Tuberculosis. Mayo Clinic Proceedings 71:221-229,1996.

8. Davis LE, Rastogi KR, Lambert LC, Skipper BJ. Tuberculous meningitis in the Southwest United States: a community-based study. Neurology 43:1775-1778, 1993.

9. Deeny JE, Walker MJ, Kibel MA, Molteno CD, Arens LJ. Tuberculous meningitis in children in the Western Cape. South African Medical Journal 68:75-78, 1985.

10. Frieden TR, Sterling T, Paplos-Mendez A, Kilburn JO, Cauthen GM, Dooleu SW. The Emergence of Drug Resistant Tuberculosis in New York City. The New England Journal of Medicine 328:521-526 1993.

11. Girgis NI, Sippel JE, Kilpatrick ME, Sanborn WR, Mikhail AI, Cross E, Erian MW, Sultan Y, Farid Z. Meningitis and Encephalitis at the Abbassia Fever Hospital, Cairo, Egypt, from 1966 to 1989. The American Journal of Tropical and Medicine Hygiene 48:97-107,1993.

12. Haas DW, Prez RMD. Mycobacterium tuberculosis. In: Mandell GL, Bennett JE, Dolin R (eds) Principles and Practice of Infectious Diseases, Churchill Levingstone, New York, p.2213-2250, 1995.

13. Jereb AJ, Klevens RM, Privett TD, Smith PJ, Crawford JT, Sharp VL, Davis BJ, Jarvis WR, Dooley SW.
Tuberculosis in Health Care Workers at a Hospital Wich na Outbreak of Multidrug-Resistant Mycobacterium tuberculosis. Archives of Internal Medicine 155:854-859,1995.

14. Kennedy DH, Fallon RJ. Tuberculous Meningitis. Journal of the American Medical Association 241:264-268, 1979.

15. Leonard JM, Des Prez RM. Meningite Tuberculosa. In: Moellering Jr RC (ed) Clínicas de Doenças Infecciosas da América do Norte. Interlivros, Rio de Janeiro, volume 4, p.761-779,1990.

16. Martins TS, Medeiros CO, Gerhardt GF. Meningite Tuberculosa (Resultados de uma Pesquisa Operacional). Jornal Brasileiro de Medicina 4:3441,1985 .

17. Molavi A, Le Frock JL. Tuberculous Meningitis. Medical Clinics of North America 69:315-331, 1985.

18. Nardy SMC. Aspectos epidemiológicos da meningite tuberculosa em menores de 15 anos de idade, na grande São Paulo, Brasil, 1982-1983. Revista de Saúde Pública 23:117-127, 1989.

19. Newton RW. Tuberculous meningitis. Archives of Disease in Childhood 70:364-366, 1994.

20. Nunes C, Gomes I, Tavares A, Melo A. Características clínicas e laboratoriais de 62 casos de meningoencefalite tuberculosa. Arquivos de Neuropsiquiatria 54:222-226, 1996.

21. Organización Panamericana de la Salud. Control de la tuberculosis: Manual sobre métodos y procedimientos para los programas integrados. Organizacion Panamericana de la Salud, Publicación Científica nำ 498, 1987.

22. Perfeito JB, Assis JL. Meningoencefalite Tuberculosa. In: Veronesi R, Focaccia R, Dietze R (eds), Doenças Infecciosas e Parasitárias. 8ª edição, Guanabara Koogan, Rio de Janeiro, p. 510-519, 1991.

23. Singh NK, Singh P, Tripathi K, Sruvastava PK, Singh DS. Prognostic Factors and Sequelae of tuberculous Meningitis in Adults. Journal of Indian Medical Assocition 83:50-53 1985.

24. Valenzuela MT, Carrasco E, Garcia P, Toro J. Analisis Retrospectivo del Prognóstico de la Meningitis Tuberculosa en Chile, en Relacion al Diagnostico Y al Tratamiento. Enfermedades Respiratorias y Cirurgia Torácica 4:124-132, 1988.

25. Wunsch Filho V, Castilho EA, Rodrigues LC, Huttly SRA. Effectiveness of BCG vaccination against tuberculous meningitis: a case-control study in São Paulo, Brazil. Bulletin of the World Health Organization 68:69-74, 1990. 
26. Zuger A, Lowy F. Tuberculosis of the Central Nervous System. In: Sheld WM, Whithey RJ, Durack DT (eds)
Infections of the Central Nervous System. Raven Press, New York, p.425-739, 1991. 